\title{
Electron rest mass and energy levels of atoms in photonic crystal medium
}

\author{
Renat Kh. Gainutdinov * Marat A. Khamadeev, and Myakzyum Kh. Salakhov
}

(Dated: July 27, 2021)

\begin{abstract}
Photonic crystals are periodic systems that consist of dielectrics with different refractive indices. They are designed to act on photons in contrast to semiconductor crystals whose periodicity affects the motion of electrons. Here we consider the interaction of an atomic electron with its own radiation field in the case when the atom is placed in air voids of a photonic crystal and is not in mechanical contact with the vibrational degrees of freedom of the dielectric host. A strong modification of this interaction from that in free space is shown to change the rest mass of the electron, and this has a significant effect on the shift of the atomic energy levels. This shift is investigated by using the example of atomic hydrogen in a high-index-contrast photonic crystal. The found effect may be of interest both from fundamental and practical points of view.
\end{abstract}

PACS numbers: 42.70.Qs, 42.50.Ct, 31.30.J-

\section{INTRODUCTION}

Since the pioneering works of Yablonovitch [1] and John [2] photonic crystals (PCs) are a major field of research. The variation of the photon density of states (DOS) being a result of a modification of the electromagnetic fields in PCs leads to quantum effects, including the coherent control of the spontaneous emission [3], the appearance of photon-atom bound states [4 7], the non-Markovian character of radiative decay [8], enhanced quantum interference effects [9], and the localization of superradiance near the photonic band edge [10]. It is important that the strong modification of the DOS in PCs may provide new insight into the fundamental problems of quantum electrodynamics (QED).

One of the most important effects of QED is the Lamb shift that originally was defined as a splitting of $2 P_{1 / 2}$ and $2 S_{1 / 2}$ levels of atomic hydrogen. This motivated many efforts devoted to the study of the Lamb shift in PCs [4, 5, 11 15]. It was found that the interaction of an atom with its own radiation field can be significantly modified in the PC medium, and as a result, can lead to very large values of the Lamb shift, as compared to the case of vacuum [14, 15]. The effect was investigated by means of the standard methods that were successfully employed for describing the Lamb shift in vacuum. However, one has to keep in mind that because of the ultraviolet (UV) divergences, in the theory of QED the Hamiltonian (Lagrangian) is only of formal importance, and knowing them is not sufficient to compute results for physical quantities. In addition, one needs to choose a renormalization scheme which regulates the integrals and subtracts the infinities. One of the key elements of the scheme is the procedure of the mass renormalization that prescribes to subtract, in solving the bound-state problem, the self-energy of a free electron from that of

*Electronic address: Renat.Gainutdinov@ksu.ru Also at Department of Physics, Kazan Federal University, 18 Kremlevskaya St, Kazan 420008, Russia. the bound electron on the basis that the electromagnetic mass of the electron must be included in its observable mass. Here we show that in the case when we deal with atoms in PCs this renormalization procedure removes not only infinities appearing in the theory but also observable contributions to physical quantities.

The origin of the problem is the fact that because of the modification of the interaction of a charged particle in the PC medium with its own radiation field, the electromagnetic mass of an electron in the PC must differ from its electromagnetic mass in vacuum, which is included into the observable mass of the electron. This means that in the PC medium the rest mass of the electron should change its value. We show that this change is observable and gives rise to a significant shift of the energy levels of an isolated atom in PCs provided it is not in mechanical contact with the vibrational degrees of freedom of the dielectric host. The effect is investigated by using the example of atomic hydrogen in a high index-contrast photonic crystal.

\section{INFLUENCE OF AN ENVIRONMENT ON THE ELECTROMAGNETIC MASS OF THE ELECTRON}

The electromagnetic mass of the electron is its selfenergy associated with the interaction of the electron with its own radiation field. Because of the UV divergences, this correction to the electron mass is infinite. The problem is solved by using the renormalization theory that implies that from the very beginning the theory is formulated in terms of the physical charge and electron mass including all radiative corrections, and correspondingly the infinite electron self-energy contributions are subtracted in computing physical quantities such as the Lamb shift in atoms. This renormalization procedure proved to be very successful in computing the QED corrections to the energy levels of isolated atoms. However, as we show below, by using the example of the atomic hydrogen in the case when atoms are removed from the vacuum and placed in an environment in which the photonic 
density of states is different from that of the vacuum, such a subtraction leads to missing an observable correction to the electron rest mass. The dominant contribution to the Lamb shift in hydrogenlike atoms is given by the onephoton (one-loop) self-energy arising from the processes in which a photon is emitted and then is reabsorbed by a bound electron [these processes are described by the diagram in Fig. [(a)] and from the processes in which the electron in its final state first appears out of vacuum together with a photon and a positron which then annihilate along with the initial electron [these processes are described by the diagram in Fig. [1(b)]. In quantum elec-
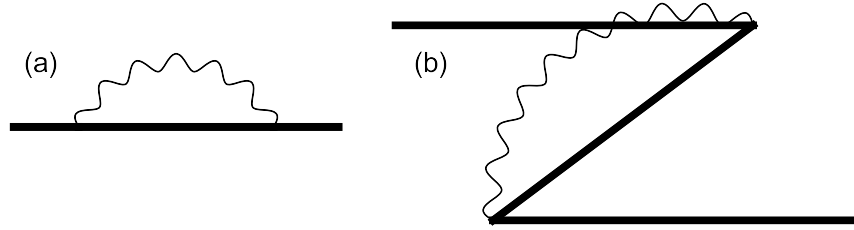

FIG. 1: The time-ordered diagrams describing the dominant contribution to the Lamb shift. The thick line denotes the electron (positron) propagating in the Coulomb field; the wavy line denotes emission and reabsorption of a virtual photon.

trodynamics the corresponding contribution to the Lamb shift is given by the term that appears in the secondorder perturbation theory, and in the Furry picture can be written as

$$
\Delta E_{n}=\left\langle n\left|H_{I} \frac{1}{E_{n}^{(0)}-H_{0}^{F}} H_{I}\right| n\right\rangle,
$$

where $H_{0}^{F}$ is the unperturbed Dirac-Coulomb Hamiltonian in the Furry picture $\left(H_{0}^{F}|n\rangle=E_{n}^{(0)}|n\rangle\right)$, and $H_{I}=\int d^{3} x \mathcal{H}_{I}(t=0, \mathbf{x})$, with $\mathcal{H}_{I}(t, \mathbf{x})$ being the interaction Hamiltonian density

$$
\mathcal{H}_{I}(t, \mathbf{x})=\frac{e}{2} A_{\mu}(t, \mathbf{x})\left[\bar{\Psi}(t, \mathbf{x}), \gamma^{\mu} \Psi(t, \mathbf{x})\right]
$$

It involves the quantized electromagnetic field $A_{\mu}(x)$ and the quantized Dirac field $\Psi(x)$. The Furry picture is a kind of interaction representation in which the unperturbed Hamiltonian $H_{0}^{F}$ is the sum of the true free Hamiltonian $H_{0}$ and the interaction Hamiltonian describing the interaction with an external field, and provides the most convenient way to solve the bound state problem in quantum electrodynamics.

Usually the self-energy correction to the energy levels of hydrogenlike atoms is calculated by dividing the integral over virtual photon energies, which is involved in (11), into a low-energy range $(k<\Lambda)$, within which one can treat the electron nonrelativistically but must take into account effects to all orders in the external field, and a high energy range $(k>\Lambda)$, within which the problem must be treated relativistically but in the lowest order in the external field. The parameter $\Lambda$ must be chosen to be much larger than the atomic binding energies, but much less than typical electron momenta (here and below we use the unit system where $\hbar=c=\varepsilon_{0}=1$ )

$$
(Z \alpha)^{2} m_{e} \ll \Lambda \ll(Z \alpha) m_{e} .
$$

Thus, the self-energy shift is the sum of two terms, the high-energy (HE) term $\Delta E_{n}^{>}$and the low-energy (LE) term $\Delta E_{n}^{<}$. In the nonrelativistic approximation we may neglect the contribution to the Lamb shift from the processes described by the diagram depicted in Fig. 1(b), and as a consequence, for $\Delta E_{n}^{<}$we get from Eq. (11) the following expression [16]:

$$
\Delta E_{n}^{<}=\frac{2 \pi \alpha}{3 m_{e}^{2}} \int_{0}^{\Lambda} \frac{d^{3} k}{2|\mathbf{k}|(2 \pi)^{3}} \sum_{m} \frac{|\langle n|\mathbf{p}| m\rangle|^{2}}{E_{n}-|\mathbf{k}|-E_{m}},
$$

where $\alpha$ is the fine-structure constant. The above prescription of the renormalization theory tells us that the self-energy of a free electron must be subtracted from the self-energy of the bound electron given by Eq. (3). The contribution to the self-energy of the free electron comes from the processes in which in time intervals between the emission and reabsorption the electron is free and does not interact with the Coulomb field. Since for a free electron only diagonal elements of the operator $\mathbf{p}^{2}$ differ from zero, the contribution to $\Delta E_{n}^{<}$given by Eq. (3) from such processes is

$$
\Delta E_{v, n}^{<}=-\frac{\Delta m_{e}}{2 m_{e}^{2}}\left\langle n\left|\mathbf{p}^{2}\right| n\right\rangle,
$$

where

$$
\Delta m_{e}=\frac{\alpha}{p^{2} \pi^{2}} \sum_{\lambda=1}^{2} \int_{0}^{\Lambda} \frac{d^{3} k}{2|\mathbf{k}|^{2}}\left|\mathbf{p} \cdot \varepsilon_{\lambda}(\mathbf{k})\right|^{2} .
$$

As follows from Eq. (4), $\Delta m_{e}$ should be regarded as a contribution to the electromagnetic mass of the electron [17]. Thus, $\Delta m_{e}$ in Eq. (4) is the electromagnetic mass of the electron and therefore must be subtracted because the mass $m_{e}$ which we deal with is the physical mass of the electron including all the radiative corrections. This subtraction yields

$\Delta E_{n}^{<}=\frac{\alpha}{6 \pi^{2} m_{e}^{2}} \int_{0}^{\Lambda} \frac{d^{3} k}{2|\mathbf{k}|^{2}} \sum_{m} \frac{|\langle n|\mathbf{p}| m\rangle|^{2}}{E_{n}-|\mathbf{k}|-E_{m}}\left(E_{n}-E_{m}\right)$.

Adding the high-energy part $\Delta E_{n}^{>}$, which is calculated by using the corresponding Feynman diagrams of quantum electrodynamics, to this term, we arrive [16] at the ordinary expression for the one-loop self-energy Lamb shift $\Delta E_{n}=\Delta E_{n}^{<}+\Delta E_{n}^{>}$in a hydrogenlike atom where the logarithmic dependence of $\Delta E_{n}^{<}$on $\Lambda$ is compensated by that of $\Delta E_{n}^{>}$.

In order to generalize the theory for describing the Lamb shift in atomic hydrogen placed in PCs, one has to take into account the influence of the PC medium on the 
propagation of virtual photons that come into play in the process of the self-interaction of the atomic electron. Correspondingly, in this case the wavy lines in Fig. 1 should describe the propagation of photons in the PC medium. Formally, carrying out the mass renormalization for the electron in the PC medium should result in the subtraction of the self-energy of the free electron modified by this medium from the modified self-energy of the bound electron, and this subtraction was used [4, 5, 11 15] in the studies of the Lamb shift in atoms placed in PCs. However, this way of solving the problem leads to missing some important contributions to energy levels from the self-interaction of atomic electrons. In fact, the renormalization theory prescribes to also add the subtracted term $\Delta m_{e}$ to the "bare" mass $m_{0}$ of the electron in order to arrive at its physical mass $m_{e}=m_{0}+\Delta m_{e}$. Here we mean one of the two approaches to renormalization. It has the merit of a clear physical interpretation, but the second approach, the method of counterterms, is the one normally used in quantum field theory. In the second approach the electron mass $m_{e}$ in the original Lagrangian is regarded as the physical mass. To cancel the contribution from the self-energy of the free electron an extra term is added to the Lagrangian that is called the mass-renormalization counterterm. The problem is that the value of the electromagnetic mass of the electron in the PC medium should differ from that in vacuum, and therefore the result of adding this electromagnetic mass to its "bare" mass will not be the physical mass. Obviously, the change of the value of the electron mass $\delta m_{p c}$ is the difference between the values of the electromagnetic masses in the PC medium $\Delta m_{p c}$ and the electromagnetic mass $\Delta m_{e}$ in vacuum

$$
\delta m_{p c}=\Delta m_{p c}-\Delta m_{e} .
$$

Thus, the influence of the PC medium on the interaction of an electron with its own radiation field results in the change in its mass: $m_{e} \rightarrow m_{p c}=m_{e}+\delta m_{p c}$. Actually the above arguments are correct for any environment in which the photonic density of states is different from that of the vacuum, and allow one to conclude that the rest mass of the electron placed in this environment should change its value.

\section{PHOTONIC CRYSTAL MEDIUM CORRECTIONS TO THE ELECTRON REST MASS}

Let us consider the problem of the change in rest mass of an electron in the PC medium in more detail. Since the behavior of photons in the PC medium differs from that in vacuum only in the optical range of frequencies, from Eq. (6) it follows that only the low-energy part of the electron electromagnetic mass is relevant for the problem. In this case the problem can be solved nonrelativistically and it is convenient to choose the Coulomb gauge that has the advantage that the radiation is completely described by the vector potential $\mathbf{A}$. In this gauge the nonrelativistic Hamiltonian for an electron in an electromagnetic field may be written in the form

$$
H_{e l}=\frac{1}{2 m_{e}}[\mathbf{p}-e \mathbf{A}(\mathbf{r})]^{2},
$$

where $\mathbf{r}$ is the position of the electron. The one-loop LE contribution $\Delta m_{e}$ to the electromagnetic mass of an electron in the Coulomb gauge takes the form

$$
\Delta m_{e}=-\frac{2 m_{e}^{2}}{\mathbf{p}^{2}} \sum_{\mathbf{p}^{\prime}} \sum_{\mathbf{k} \varepsilon_{\lambda}} \frac{\left\langle\mathbf{p}\left|H_{I}\right| \mathbf{p}^{\prime} ; \mathbf{k}, \varepsilon_{\lambda}\right\rangle\left\langle\mathbf{p}^{\prime} ; \mathbf{k}, \varepsilon_{\lambda}\left|H_{I}\right| \mathbf{p}\right\rangle}{\frac{\mathbf{p}^{2}}{2 m_{e}}-\frac{\mathbf{p}^{\prime 2}}{2 m_{e}}-|\mathbf{k}|}
$$

where $H_{I}=-\frac{e}{m_{e}} \mathbf{p} \cdot \mathbf{A}$ and $\left|\mathbf{p} ; \mathbf{k}, \varepsilon_{\lambda}\right\rangle$ is a state with an electron with momentum $\mathbf{p}$ and a photon with momentum $\mathbf{k}$ and polarization vector $\varepsilon_{\lambda}$. Here and below, in order to deal with states of norm 1, we discretize the continuum by enclosing the particles in a cubic box of volume $V$, and by imposing periodic boundary conditions to obtain states having the same spatial dependence as the states $|\mathbf{p}\rangle$. The final results for physical quantities must not depend on $V$ provided it is large enough. Since in our investigations we deal with the electrodynamics within a $\mathrm{PC}$ it is natural to use its volume as the normalization volume $V$.

Obviously, in describing the low-energy part of the electron self-energy in the PC medium it is especially important to take into account the Bloch structure of the photon states that arises because of the periodicity of dielectric function $\varepsilon(\mathbf{r})$. This structure means that the photon states can be expanded in a set of Bloch states $|\mathbf{k} n\rangle$, which can be obtained by means of the plane-wave expansion method [18]. By introducing the operators $\hat{a}_{\mathbf{k} n}^{\dagger}$ and $\hat{a}_{\mathbf{k} n}$ that describe the creation and annihilation of the photon in the state $|\mathbf{k} n\rangle$ respectively $\left(\hat{a}_{\mathbf{k} n}^{\dagger}|0\rangle=|\mathbf{k} n\rangle\right.$ and $\hat{a}_{\mathbf{k} n}|\mathbf{k} n\rangle=|0\rangle$ ), we can construct a modified "free" Hamiltonian $H_{0}^{f}=\sum_{\mathbf{k n}} \omega_{\mathbf{k n}} \hat{a}_{\mathbf{k} n}^{\dagger} \hat{a}_{\mathbf{k} n}$ and a quantized vector potential 


$$
\mathbf{A}_{p c}(\mathbf{r}, t)=\sum_{\mathbf{k} n}\left[\mathcal{A}_{\mathbf{k} n}(\mathbf{r}) \hat{a}_{\mathbf{k} n} e^{-i \omega_{\mathbf{k n}} t}+\mathcal{A}_{\mathbf{k} n}^{*}(\mathbf{r}) \hat{a}_{\mathbf{k} n}^{\dagger} e^{i \omega_{\mathbf{k n}} t}\right],
$$

where $\mathcal{A}_{\mathbf{k} n}(\mathbf{r})=\sqrt{1 / V \omega_{\mathbf{k} n}} \mathbf{E}_{\mathbf{k} n}(\mathbf{r})$ with $\mathbf{E}_{\mathbf{k} n}(\mathbf{r})$ being the Bloch eigenfunctions satisfying the following orthonormality condition

$$
\int_{V} d^{3} r \varepsilon(\mathbf{r}) \mathbf{E}_{\mathbf{k} n}(\mathbf{r}) \mathbf{E}_{\mathbf{k}^{\prime} n^{\prime}}^{*}(\mathbf{r})=V \delta_{\mathbf{k k}^{\prime}} \delta_{n n^{\prime}} .
$$

In this way we actually arrive at a modified Furry picture, in which not only the interaction of an electron with the Coulomb field but also the interaction of photons with the PC medium is taken into account from the very beginning. Correspondingly, in the interaction
Hamiltonian (7) the quantized vector potential describing the free electromagnetic field in the PC medium should be replaced with $A_{p c}(\mathbf{r}, \mathbf{t})$ defined by Eq. (9). With the vector potential defined in this way the expression for the Hamiltonian (7) is transformed to

$$
H_{e l}^{p c}=\frac{1}{2 m_{e}}\left[\mathbf{p}-e \mathbf{A}_{p c}(\mathbf{r})\right]^{2} .
$$

Correspondingly, the expression for the LE part of the electromagnetic mass of the electron in the PC medium takes the form

$$
\Delta m_{p c}=-\frac{2 m_{e}^{2}}{\mathbf{p}^{2}} \sum_{\mathbf{p}^{\prime}} \sum_{\mathbf{k} n}^{\omega_{\mathbf{k} n}<\Lambda} \frac{\left\langle\mathbf{p}\left|H_{I}^{p c}\right| \mathbf{p}^{\prime} ; \mathbf{k}, n\right\rangle\left\langle\mathbf{p}^{\prime} ; \mathbf{k}, n\left|H_{I}^{p c}\right| \mathbf{p}\right\rangle}{\frac{\mathbf{p}^{2}}{2 m_{e}}-\frac{\mathbf{p}^{\prime 2}}{2 m_{e}}-\omega_{\mathbf{k} n}}
$$

Taking into account that for the above reason only the LE parts of $\Delta m_{e}$ and $\Delta m_{p c}$ give a contribution to $\delta m_{p c}$ defined by Eq. (6), from this equation we get

$$
\delta m_{p c}=-\frac{2 m_{e}^{2}}{\mathbf{p}^{2}}\left(\sum_{\mathbf{p}^{\prime}} \sum_{\mathbf{k} n}^{\omega_{\mathbf{k} n}<\Lambda} \frac{\left\langle\mathbf{p}\left|H_{I}^{p c}\right| \mathbf{p}^{\prime} ; \mathbf{k}, n\right\rangle\left\langle\mathbf{p}^{\prime} ; \mathbf{k}, n\left|H_{I}^{p c}\right| \mathbf{p}\right\rangle}{\frac{\mathbf{p}^{2}}{2 m_{e}}-\frac{\mathbf{p}^{\prime 2}}{2 m_{e}}-\omega_{\mathbf{k} n}}-\sum_{\mathbf{p}^{\prime}} \sum_{\mathbf{k} \varepsilon_{\lambda}}^{|\mathbf{k}|<\Lambda} \frac{\left\langle\mathbf{p}\left|H_{I}\right| \mathbf{p}^{\prime} ; \mathbf{k}, \varepsilon_{\lambda}\right\rangle\left\langle\mathbf{p}^{\prime} ; \mathbf{k}, \varepsilon_{\lambda}\left|H_{I}\right| \mathbf{p}\right\rangle}{\frac{\mathbf{p}^{2}}{2 m_{e}}-\frac{\mathbf{p}^{\prime 2}}{2 m_{e}}-|\mathbf{k}|}\right) .
$$

Here the cutoff $\Lambda$ in expressions for $\Delta m_{e}$ and $\Delta m_{p c}$ is removed because virtual $\mathrm{HE}$ photons emitted by an electron in the PC medium propagate as if they were in vacuum. For this reason the contributions to the first and second terms on the right-hand part of Eq. (13) that come from the self-interaction processes involving highenergy virtual photons must compensate each other. The matrix element $\left\langle\mathbf{p}^{\prime} ; \mathbf{k}, n\left|H_{I}^{p c}\right| \mathbf{p}\right\rangle$ of the interaction Hamiltonian $H_{I}^{p c}=-\frac{e}{m_{e}} \mathbf{p} \cdot \mathbf{A}_{p c}$ can be represented in the form

$$
\left\langle\mathbf{p}^{\prime} ; \mathbf{k}, n\left|H_{I}^{p c}\right| \mathbf{p}\right\rangle=-\frac{e}{m_{e}} \int d^{3} r \Psi_{\mathbf{p}^{\prime}}^{*}(\mathbf{r})\left[-i \nabla_{\mathbf{r}} \mathbf{A}_{\mathbf{k} n}(\mathbf{r})\right] \Psi_{\mathbf{p}}(\mathbf{r})=\frac{e}{m_{e} V^{3 / 2} \sqrt{\omega_{\mathbf{k} n}}} \int d^{3} r e^{-i \mathbf{p}^{\prime} \mathbf{r}}\left[i \nabla_{\mathbf{r}} \mathbf{E}_{\mathbf{k} n}(\mathbf{r})\right] e^{i \mathbf{p r}}
$$

with $\Psi_{\mathbf{p}}(\mathbf{r})$ being the normalized wave function of the electron state $\Psi_{\mathbf{p}}(\mathbf{r})=\langle\mathbf{r} \mid \mathbf{p}\rangle$. Here we have taken into account that $\Psi_{\mathbf{p}}=e^{i \mathbf{p r}} / \sqrt{V}$ for $\mathbf{r} \in V$ and $\Psi_{\mathbf{p}}=0$ for $\mathbf{r} \notin V$. Taking also into account that $\mathbf{E}_{\mathbf{k} n}(\mathbf{r})$ can be expanded as

$$
\mathbf{E}_{\mathbf{k} n}(\mathbf{r})=\sum_{\mathbf{G}} \mathbf{E}_{\mathbf{k} n}(\mathbf{G}) e^{i(\mathbf{k}+\mathbf{G}) \cdot \mathbf{r}}
$$

with $\mathbf{G}$ being the reciprocal lattice vector of the photonic $\operatorname{crystal}\left(\mathbf{G}=N_{1} \mathbf{b}_{1}+N_{2} \mathbf{b}_{2}+N_{3} \mathbf{b}_{3}\right.$, where $\mathbf{b}_{i}$ are primitive basis vectors of a reciprocal lattice), for $\left\langle\mathbf{p}^{\prime} ; \mathbf{k}, n\left|H_{I}^{p c}\right| \mathbf{p}\right\rangle$ we get

$$
\left\langle\mathbf{p}^{\prime} ; \mathbf{k}, n\left|H_{I}^{p c}\right| \mathbf{p}\right\rangle=-\frac{e}{m} \frac{1}{\sqrt{V \omega_{\mathbf{k} n}}} \sum_{\mathbf{G}} \mathbf{p} \cdot \mathbf{E}_{\mathbf{k} n}(\mathbf{G}) \delta_{\mathbf{p}, \mathbf{q}},
$$

with $\mathbf{q}=\mathbf{p}^{\prime}+\mathbf{k}+\mathbf{G}$. In the same way, for $\left\langle\mathbf{p}\left|H_{I}^{p c}\right| \mathbf{p}^{\prime} ; \mathbf{k}, n\right\rangle$ we find

$$
\left\langle\mathbf{p}\left|H_{I}^{p c}\right| \mathbf{p}^{\prime} ; \mathbf{k}, n\right\rangle=-\frac{e}{m_{e}} \frac{1}{\sqrt{V \omega_{\mathbf{k} n}}} \sum_{\mathbf{G}} \mathbf{p} \cdot \mathbf{E}_{\mathbf{k} n}^{*}(\mathbf{G}) \delta_{\mathbf{p}, \mathbf{q}}
$$


Correspondingly, for the matrix elements of the interaction Hamiltonian in the free space, we have

$$
\begin{aligned}
\left\langle\mathbf{p}^{\prime} ; \mathbf{k}, n\left|H_{I}\right| \mathbf{p}\right\rangle & =-\frac{e}{m_{e}} \frac{1}{\sqrt{2 V|\mathbf{k}|}} \sum_{\lambda} \mathbf{p} \cdot \varepsilon_{\lambda}(\mathbf{k}) \delta_{\mathbf{p}, \mathbf{q}}, \\
\left\langle\mathbf{p}\left|H_{I}\right| \mathbf{p}^{\prime} ; \mathbf{k}, n\right\rangle & =-\frac{e}{m_{e}} \frac{1}{\sqrt{2 V|\mathbf{k}|}} \sum_{\lambda} \mathbf{p} \cdot \varepsilon_{\lambda}(\mathbf{k}) \delta_{\mathbf{p}, \mathbf{q}},
\end{aligned}
$$

with $\mathbf{q}=\mathbf{p}^{\prime}+\mathbf{k}$. Substituting these matrix elements of interaction Hamiltonians $H_{I}^{p c}$ and $H_{I}$ into Eq. (13) yields

$$
\delta m_{p c}=-\frac{2 e^{2}}{\mathbf{p}^{2} V}\left(\sum_{\mathbf{G}} \sum_{\mathbf{k} n} \frac{1}{\omega_{\mathbf{k} n}} \frac{\left|\mathbf{p} \cdot \mathbf{E}_{\mathbf{k} n}(\mathbf{G})\right|^{2}}{\frac{\mathbf{p}^{2}}{2 m_{e}}-\frac{(\mathbf{p}-\mathbf{k}-\mathbf{G})^{2}}{2 m_{e}}-\omega_{\mathbf{k} n}}-\sum_{\mathbf{k}} \sum_{\lambda=1}^{2} \frac{1}{2|\mathbf{k}|} \frac{\left|\mathbf{p} \cdot \varepsilon_{\lambda}(\mathbf{k})\right|^{2}}{\frac{\mathbf{p}^{2}}{2 m_{e}}-\frac{(\mathbf{p}-\mathbf{k})^{2}}{2 m_{e}}-|\mathbf{k}|}\right) .
$$

Electrons in air voids of a PC mainly are atomic electrons. In the case of atomic hydrogen the momentum of the atomic electron is of order $\alpha m_{e}$. In this case $-\omega$ is the predominant term in the denominator of Eq. (14), and hence this equation can be rewritten in the form

$$
\delta m_{p c}=\frac{2 e^{2}}{\mathbf{p}^{2} V}\left(\sum_{\mathbf{G}} \sum_{\mathbf{k} n} \frac{\left|\mathbf{p} \cdot \mathbf{E}_{\mathbf{k} n}(\mathbf{G})\right|^{2}}{\omega_{\mathbf{k} n}^{2}}-\sum_{\mathbf{k}} \sum_{\lambda=1}^{2} \frac{\left|\mathbf{p} \cdot \varepsilon_{\lambda}(\mathbf{k})\right|^{2}}{2 \mathbf{k}^{2}}\right) .
$$

Now in the expression of $\delta m_{p c}$ we can replace the discrete sums by integrals $\sum_{\mathbf{k} n} \rightarrow \frac{V}{(2 \pi)^{3}} \sum_{n} \int d^{3} k$ and $\sum_{\mathbf{k}} \rightarrow$ $\frac{V}{(2 \pi)^{3}} \int d^{3} k$. In this way we get

$$
\delta m_{p c}=\frac{\alpha}{\pi^{2}}\left[\sum_{n} \int_{F B Z} \frac{d^{3} k}{\omega_{\mathbf{k} n}^{2}} \sum_{\mathbf{G}}\left|\hat{\mathbf{p}} \cdot \mathbf{E}_{\mathbf{k} n}(\mathbf{G})\right|^{2}-\int \frac{d^{3} k}{2 \mathbf{k}^{2}} \sum_{\lambda=1}^{2}\left|\hat{\mathbf{p}} \cdot \varepsilon_{\lambda}(\mathbf{k})\right|^{2}\right]
$$

with $\hat{\mathbf{p}}=\mathbf{p} /|\mathbf{p}|$. Thus, in contrast to the Lamb shift, the PC medium correction to the electron mass does not depend on the position of the electron in a PC's air void. At the same time, this correction depends on the direction unit vector $\hat{\mathbf{p}}=\mathbf{p} /|\mathbf{p}|$ of the electron momentum. In order to describe the "mean" correction, we may average $\hat{\mathbf{p}}$ over all solid angles by assuming that this vector is randomly orientated in space

$$
\delta m_{p c}^{\Omega} \equiv \frac{1}{4 \pi} \int d \Omega \delta m_{p c}=\frac{\alpha}{3 \pi^{2}}\left[\sum_{n} \int_{F B Z} \frac{d^{3} k}{\omega_{\mathbf{k} n}^{2}} \sum_{\mathbf{G}}\left|\mathbf{E}_{\mathbf{k} n}(\mathbf{G})\right|^{2}-\int \frac{d^{3} k}{\mathbf{k}^{2}}\right] .
$$

The fact that the PC medium correction to the electron mass does not depend on the position of the electron in the PC's air void, allows us to represent the correction in the form

$$
\delta m_{p c}^{\Omega}=\frac{4 \alpha}{3 \pi} \int d \omega \frac{N(\omega)-\omega^{2}}{\omega^{2}}
$$

where $N(\omega)=N_{D O S}(\omega) D(\omega), N_{D O S}(\omega)$ is the photon density of states

$$
N_{D O S}(\omega)=\frac{1}{4 \pi} \sum_{n} \int_{F B Z} d^{3} k \delta\left(\omega-\omega_{\mathbf{k} n}\right)
$$

and

$$
D(\omega)=\sum_{\mathbf{G}}\left|\mathbf{E}_{\mathbf{k} n}(\mathbf{G})\right|_{\mid \omega_{\mathbf{k} n}=\omega}^{2} .
$$

The DOS describes the number of states per interval of energy at each energy level that are available to be occupied, and in vacuum is equal to $\omega^{2}$. In the limit $\varepsilon(\mathbf{r}) \rightarrow 1$ the right-hand part of Eq. (18) must vanish. It is easy to show that this is actually the case. In fact, since

$$
\sum_{\mathbf{G}}\left|\mathbf{E}_{\mathbf{k} n}(\mathbf{G})\right|^{2}=\frac{1}{V} \int_{V} d^{3} r\left|\mathbf{E}_{\mathbf{k} n}(\mathbf{r})\right|^{2},
$$

from Eq. (10) it follows that in the case when $\varepsilon(\mathbf{r})$ approaches 1, $\sum_{\mathbf{G}}\left|\mathbf{E}_{\mathbf{k} n}(\mathbf{G})\right|^{2}$ approaches 1 as well. Because in this case $D(\omega) \approx 1$ and hence $N(\omega) \approx N_{D O S}(\omega)$, the right-hand part of Eq. (18) becomes equal to zero. Among other things this serves as evidence of the correctness of the orthonormality condition for $\mathbf{E}_{\mathbf{k} n}(\mathbf{r})$ that determine the electromagnetic field in a PC via Eq. (9) 
Equation (18) establishes the connection between the PC medium correction to the electron mass and the DOS in a PC. For a given PC the DOS as well as the function $D(\omega)$ can be calculated numerically. However, in order to understand the dependence of the shift of the rest mass of the electron on the DOS in a $\mathrm{PC}$, it is reasonable to use a model DOS which could recapture the basic features of PCs. In Ref. [12], for example, a model DOS was proposed which recaptures the basic quantitative features of a pseudogap, and has the form

$$
N_{D O S}(\omega)=\omega^{2}\left[1-h \exp \left(-\frac{\left(\omega-\omega_{0}\right)^{2}}{\sigma^{2}}\right)\right] .
$$

However, as it is easy to see, the difference between the model DOS and that in vacuum $\omega^{2}$ decreases exponentially as the frequency $\omega$ increases or decreases from $\omega_{0}$. This means that the behavior of photons in the medium of such a PC differs from that in free space only in the frequency range around $\omega_{0}$ with the width of the order of $\sigma$. In an actual PC structure the DOS differs from that in free space in a much wider frequency region in which the PC medium may be approximately treated [13] as an effective homogeneous medium with an average dielectric constant $\bar{\varepsilon}=\varepsilon \cdot f+(1-f)$ where $\varepsilon$ is the dielectric constant of the host material and $f$ is the dielectric fraction in the PC.

These features of the PC medium are recaptured, for example, by the following model function $N(\omega)$ :

$$
N(\omega)=\omega^{2} n_{\text {eff }}^{3}\left[1-h \exp \left(-\frac{\left(\omega-\omega_{0}\right)^{2}}{\sigma^{2}}\right)\right] F(\omega),
$$

where the factor $F(\omega)=n_{\text {eff }}^{-3}+(1-$ $\left.n_{\text {eff }}^{-3}\right) /(\exp \{(\omega-\mu) / \tau\}+1)$ with $n_{\text {eff }} \equiv \sqrt{\bar{\varepsilon}}$ allows one to take into account that at high enough photon energies $N(\omega)$ must approach the free-space DOS (Fig. 2).

The results of our calculations of $\delta m_{p c}^{\Omega}$ displayed in Figs. 3(a) and 3(b) show that the shift of the rest energy of the electron in the PC medium is insensitive to the values of the model parameters $h$ and $\sigma$ characterizing the pseudogap. This means that in the one-loop approximation the contribution to the rest electron mass that comes from the virtual photons with frequencies contained within the pseudogap is negligible, and the effect depends mainly on the behavior of the DOS at much higher frequencies. For given values of the refractive index of the host dielectric and the filling fraction $f$, this behavior is mainly determined by the parameter $\mu$, whose value is chosen for the model DOS to approach the freespace DOS at frequencies higher than the upper bound $\omega_{o p}$ of the optical frequency region. For the parameters presented in the caption to Figs. 3(a) and 3(b), our calculations have given $\delta m_{p c}^{\Omega}=2.4 \cdot 10^{-6} m_{e}$.

The change in the electron mass in a PC means that the energy of the electron with the momentum $\mathbf{p}$ that in

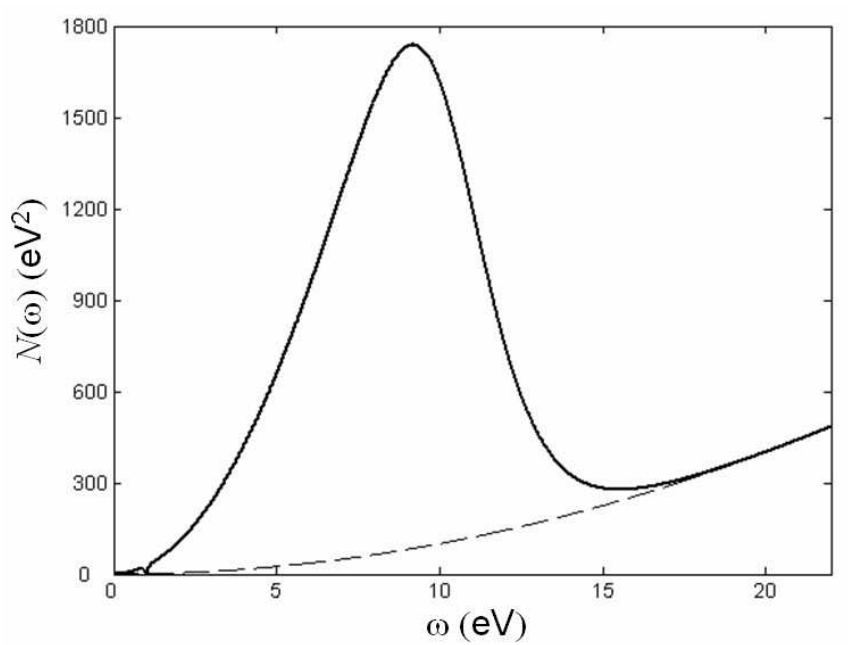

FIG. 2: The model $N(\omega)$ determined by Eq. (20) with $n_{\text {eff }}=$ $3, h=0.96, \sigma=0.07 \mathrm{eV}, \mu=15 \mathrm{eV}, \tau=0.01 \mathrm{eV}$, and $\omega_{0}=1$ $\mathrm{eV}$. The dashed line denotes the free-space DOS.

free space is (in the nonrelativistic limit) $E_{\mathbf{p}}=m_{e}+\frac{\mathbf{p}^{2}}{2 m_{e}}$ is changed to

$$
E_{\mathbf{p}}=\left(m_{e}+\delta m_{p c}\right)+\frac{\mathbf{p}^{2}}{2\left(m_{e}+\delta m_{p c}\right)} .
$$

As follows from Eq. (21), the mass correction $\delta m_{p c}$ depends on the orientation of the electron momentum in a PC. Here it should be noted that we have derived the mass correction from the contribution to the electron selfenergy of the form $-\frac{\delta m_{p c}}{m_{e}} \frac{\mathbf{p}^{2}}{2 m_{e}}$, while there is the contribution to the self-energy that does not depend on the electron momentum and directly determines the correction $\delta m_{p c}$ to the rest energy. The reason for this is that in describing the LE part of the self-energy we used the nonrelativistic Hamiltonian (11). The free part of this Hamiltonian is $\frac{\mathbf{p}^{2}}{2 m_{e}}$, and the energy is understand as the difference between the total energy and the rest energy $\epsilon_{\mathbf{p}}=E_{\mathbf{p}}-m_{e}$. Thus, the self-interaction correction to the electron energy obtained in this way does not contain the correction to the rest energy, and for $\epsilon_{\mathbf{p}}$ in the PC medium we have $\epsilon_{\mathbf{p}}=\frac{\mathbf{p}^{2}}{2\left(m_{e}+\delta m_{p c}\right)}$. However, in order to determine the total energy we must add the rest energy $m_{e}$ supplemented by the correction $\delta m_{p c}$. In this way we arrive at Eq. (21). It should be noted that the mass dependence on orientation of the electron momentum in the PC is not surprising. It is a consequence of anisotropy of the crystal. In solid-state physics, crystal anisotropies result in the fact that the effective mass of an electron depends on direction of the electron momentum with respect to the crystal axes.

Obviously the change in the electron mass gives rise to the shift of the energy levels of atoms. Let us consider the effect by using the example of the atomic hydrogen. In the approximation where the nucleus is assumed to be 

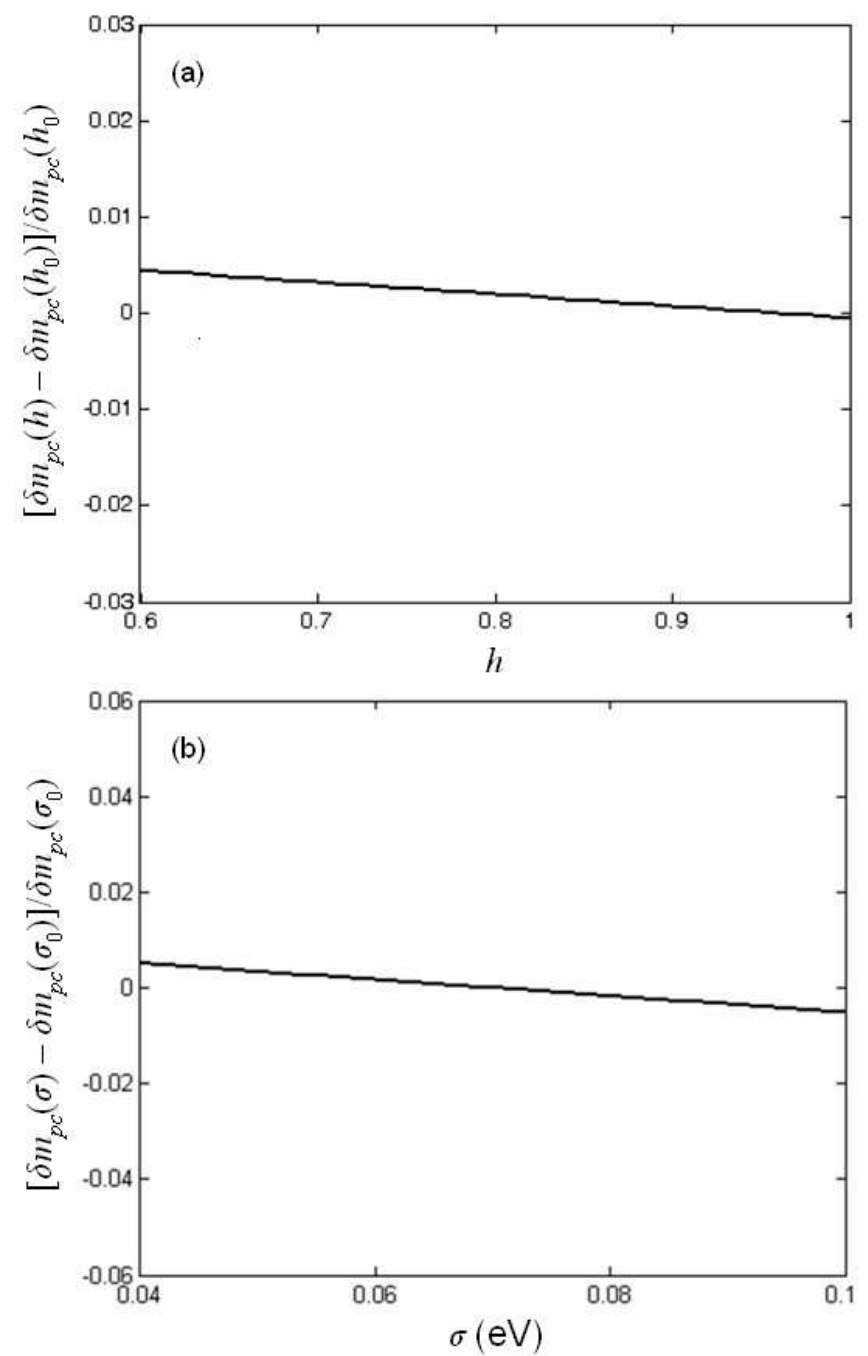

FIG. 3: The dependence of the correction $\delta m_{p c}$ of the electron rest mass in the PC medium on the values of the parameters (a) $h$ and (b) $\sigma$ characterizing the pseudogap for $n_{\text {eff }}=3$, $\mu=15 \mathrm{eV}, \tau=0.01 \mathrm{eV}, \omega_{0}=1 \mathrm{eV}, h_{0}=0.96, \sigma_{0}=0.07 \mathrm{eV}$, $\delta m_{p c}\left(h_{0}, \sigma_{0}\right)=2.4 \cdot 10^{-6} m_{e}$. Here $\delta m_{p c}$ is the mean value of the electron mass correction defined by Eq. (17)

a point and infinitely massive the energy levels of atomic hydrogen in the photonic crystal medium are given by the solution of the Dirac equation for the energy eigenvalues. For the energy of the atomic state $|n, j, l, m\rangle$, we have $E_{n j}=m_{e} R_{n j}$, with

$R_{n j}=\left[1+\left(\frac{\alpha}{n-(j+1 / 2)+\sqrt{(j+1 / 2)^{2}-\alpha^{2}}}\right)^{2}\right]^{-1 / 2}$

where the electron rest mass in vacuum is replaced by the mean mass correction $\left\langle\delta m_{p c}\right\rangle$ that is determined as

$$
\left\langle\delta m_{p c}\right\rangle=\int d^{3} p \Psi_{n j l m}^{*}(\mathbf{p}) \delta m_{p c}(\hat{\mathbf{p}}) \Psi_{n j l m}(\mathbf{p}) .
$$

Thus, the change in the rest mass of the electron in the hydrogen atom placed in a $\mathrm{PC}$ gives rise to the following shift of the energy levels

$$
\delta E_{n j}^{p c}=\left\langle\delta m_{p c}\right\rangle R_{n j} .
$$

Here we do not take into account the corrections that appear because of the modification of the Lamb shift caused by the change in the electron rest mass. In other words, the shift $\delta E_{n j}^{p c}$ of an energy level of atomic hydrogen in the $\mathrm{PC}$ medium equals its free-space value multiplied by the ratio $\left\langle\delta m_{p c}\right\rangle / m_{e}$. And in the approximation, where the atomic nucleus is assumed to be infinity massive, this is the case for any atom. This is because in this approximation there is only one energy scale that is given by the electron mass, and as a consequence, the energy of an atomic state is the electron mass multiplied by some dimensionless factor.

The energy of the hydrogen state in free space $|a\rangle=$ $|n, j, l, m\rangle$ may be written as $E_{a}=m_{e}+\epsilon_{a}$ with $\epsilon_{a}=$ $-\frac{1}{2} \frac{\alpha^{2} m_{e}}{n^{2}}+\mathrm{O}\left(\alpha^{4}\right)$. Here the rest energy part of $E_{a}$ is distinguished. The frequency $\omega_{a b}$ of the transition between the state $|a\rangle$ and the state $|b\rangle=\left|n^{\prime}, j^{\prime}, l^{\prime}, m^{\prime}\right\rangle$ is given by

$$
\omega_{a b}=\epsilon_{a}-\epsilon_{b} .
$$

The transition frequency $\omega_{a b}$ is equal to $\epsilon_{a}-\epsilon_{b}$ because the rest energy contributions are the same for both the states. The situation is dramatically changed in the case when the atom is placed in the PC medium. As we have seen, in this case the rest energy part of the total energy of the bound electron depends on the orbital angular momentum and the angular momentum $z$ component $m$. As a result, the rest energy parts of the total energies of the states $|a\rangle$ and $|b\rangle$ make a contribution to the transition frequency

$$
\omega_{a b}^{p c}=\left\langle\delta m_{p c}\right\rangle_{a}-\left\langle\delta m_{p c}\right\rangle_{b}+\epsilon_{a}^{p c}-\epsilon_{b}^{p c} .
$$

Thus, in contrast to the free-space case, in the case of the PC medium the rest energy parts of the total energies of the states $|a\rangle$ and $|b\rangle$ make the contribution to the frequency of the transition between these states. Moreover, the difference between $\left\langle\delta m_{p c}\right\rangle_{a}$ and $\left\langle\delta m_{p c}\right\rangle_{b}$ makes a predominant contribution to the $\mathrm{PC}$ correction $\delta \omega_{a b}^{p c}$ to the transition frequency

$$
\delta \omega_{a b}^{p c}=\left(\left\langle\delta m_{p c}\right\rangle_{a}-\left\langle\delta m_{p c}\right\rangle_{b}\right)\left(1+\mathrm{O}\left(\alpha^{4}\right)\right)
$$

provided $l_{a} \neq l_{b}$ and/or $m_{a} \neq m_{b}$. Such a surprising appearance of the contribution from the electron rest energy to the atom transition frequencies in the case when the atom is placed in the $\mathrm{PC}$ medium gives rise to the fact that the corrections to these frequencies can be very significant. For example, for all $S$ states, $\left\langle\delta m_{p c}\right\rangle_{n S}$ coincide with $\delta m_{p c}^{\Omega}$, which is determined by Eq. (18), and in our model is found to be $2.4 \times 10^{-6} m_{e}$. The value of the $\left\langle\delta m_{p c}\right\rangle$ in $P$ states should be different but of the same order of magnitude. Thus, the PC medium correction to the frequencies of the transitions between the $S$ and $P$ states should be of order $10^{-6} m_{e}$. 


\section{OUTLOOK}

We have shown that in the photonic crystal medium a quantum electrodynamical effect of a new type takes place. The atoms placed in a photonic crystal may be regarded as atoms in free space, and as a result, they must have the ordinary line optical spectrum. Photonic crystal medium affects only the self-radiation field of these atoms. Unlike the free-space case in the photonic crystal medium the interaction of an atomic electron with its own radiation field that contributes to the mass manifests itself explicitly and this gives rise to the change in its mass. We have derived Eq. (16) that allows one to calculate the mass correction $\delta m_{p c}$ for a given PC. From this equation it follows that $\delta m_{p c}$ is independent of the position of the electron in the PC voids but depends on the direction of the electron momentum with respect to the photonic crystal axes. This mass dependence on direction has a significant effect on the structure of the atomic energy levels because it give rise to the fact that the mean PC medium correction $\delta m_{p c}$ in states with different orbital angular momenta and/or angular momentum $z$ components are different. This in turn results in the appearance of the term $\left\langle\delta m_{p c}\right\rangle_{a}-\left\langle\delta m_{p c}\right\rangle_{b}$ in the expression (24) for the atomic transition frequency $\omega_{a b}$. Thus, despite that the modification of the interaction of the electron with its own radiation field in the $\mathrm{PC}$ medium gives rise to relatively small corrections to the rest electron mass (in our model they are of order $10^{-6} m_{e}$ ), it results in the appear- ance of the term in the expressions for atomic transition frequencies that is absent in the free-space case. This terms makes the contribution to $\omega_{a b}$ of order $10^{-6} m_{e}$, while the transition frequency in free space determined by Eq. (23) is given by $\omega_{a b}=\frac{\alpha^{2}}{2} m_{e}\left(\frac{1}{n_{b}^{2}}-\frac{1}{n_{a}^{2}}\right)+\mathrm{O}\left(\alpha^{4}\right)$. Thus, the shifts of the energy levels of the atom in the PC medium actually may be comparable to the atomic transition frequency in free space, and for this the modification of the electromagnetic field in this medium need not to be extraordinary. This provides a way to drive the structure of the atomic energy levels. In this way, in particular, light sources with the line spectrum of a new type could be developed. Every spectral line of such sources could be shifted in a wide range by changing properties of a photonic crystal. The change in the rest mass of an electron in the $\mathrm{PC}$ medium gives rise also to the change of its magnetic properties. The magnetic moment $\mu$ of an electron changes its value in the PC medium on the value $\delta \mu_{p c}=-\mu \delta m_{p c} / m_{e}$, where $\mu$ is the electron magnetic moment in vacuum. This is important in view of the observation of the effect under study as well as its applications. It is also important that the mass correction $\delta m_{p c}$ carries valuable information about the electron self-interaction that in solving other QED problem is hidden in the regularization and renormalization procedure. This gives us the hope that experimental investigations of the predicted effect might have seen beyond the physics that is described by the QED renormalization theory.
[1] E. Yablonovitch, Phys. Rev. Lett. 58, 2059 (1987).

[2] S. John, Phys. Rev. Lett. 58, 2486 (1987).

[3] T. Quang, M. Woldeyohannes, S. John, G. S. Agarwal, Phys. Rev. Lett. 79, 5238 (1997).

[4] S. John, J. Wang, Phys. Rev. Lett. 64, 2418 (1990).

[5] S. John, J. Wang, Phys. Rev. B 43, 12772 (1991).

[6] S. Bay, P. Lambropoulos, K. Mölmer, Phys. Rev. Lett. 79, 2654 (1997).

[7] S. Bay, P. Lambropoulos, K. Mölmer, Phys. Rev. A 55, 1485 (1997).

[8] K. Busch, N. Vats, S. John, B. C. Sanders, Phys. Rev. E 62, 4251 (2000).

[9] S. Y. Zhu, H. Chen, H. Huang, Phys. Rev. Lett. 79, 205 (1997).

[10] S. John and T. Quang, Phys. Rev. Lett. 74, 3419 (1995).

[11] S. Y. Zhu, Y. Yang, H. Chen, H. Zheng, and M. S. Zubairy, Phys. Rev. Lett. 84, 2136 (2000).

[12] N. Vats, S. John, and K. Busch, Phys. Rev. A 65, 043808 (2002).

[13] Z.Y. Li and Y. Xia, Phys. Rev. B 63, 121305(R) (2001).

[14] X.-H. Wang, Y. S. Kivshar, and B.-Y. Gu, Phys. Rev.
Lett. 93, 073901 (2004).

[15] X.-H. Wang, Y. S. Kivshar, and B.-Y. Gu, Science and Technology of Advanced Materials 6, 814 (2005).

[16] J. D. Bjorken, S. D. Drell, Relativistic Quantum Mechanics (McGraw-Hill, New York, 1964), Vol. 1.

[17] S. S. Schweber, An Introduction to Relativistic Quantum Field Theory (Dover, New York, 2005).

[18] K. Sakoda, Optical Properties of Photonic Crystals (Springer, Berlin, 2001).

\section{Acknowledgments}

This study was supported by Grant No. NSh5289.2010.2 of the President of the Russian Federation for Support of Leading Scientific Schools and by the Federal Target Program "Research and scientific-pedagogical cadres of innovative Russia", Grant No. 02.740.11.0428. 